Pacific Journal of Mathematics

INTERSECTIONS OF LEAST AREA SURFACES 


\title{
INTERSECTIONS OF LEAST AREA SURFACES
}

\author{
Joel Hass
}

\begin{abstract}
It is shown that hyperbolic 3-space contains an embedded curve $\gamma$ with the property that any least area disk bounded by $\gamma$ must intersect $P$. It follows that least area surfaces in 3-manifolds sometimes intersect more than topologically necessary.
\end{abstract}

Least area surfaces tend to intersect as little as possible, making them useful tools in the study of 3-dimensional manifolds. This paper will present a somewhat surprising phenomenon of excess intersection between least area surfaces in Riemannian 3-manifolds, showing that there is a limit to the generally correct philosophy that "least area surfaces intersect least" [M-Y], [F-H-S].

A plane is said to have least area if any subdisk of the plane is a solution of the Plateau problem for its boundary, so that no disk with the same boundary has less area. We will give an example of an embedded least area plane $P$ and an embedded curve $\gamma$ lying on one side of $P$ such that any least area disk $D$ which solves the Plateau problem for $\gamma$ must intersect $P$. This contrasts with the situation where the disk is embedded; an embedded least area disk is always disjoint from $P$ [M-Y]. There is no topological necessity for an intersection between $D$ and $P$, since by finding a large disk $E$ on $P$ containing $D \cap P$ and contracting $D \cap P$ within $E$, one can push off slightly to obtain a disk bounded by $\gamma$ and missing $P$. Thus the example shows that least area surfaces can intersect more than is topologically necessary. The example also shows that the "cut and paste" technique developed by Papakyriakopoulos $[\mathbf{P}]$ and applied by Meeks-Yau [M-Y] in the proofs of the geometric Sphere Theorem and geometric Dehn's Lemma will not extend to the setting of immersed surfaces.

THEOREM 1. There is an embedded least area plane $P$ in hyperbolic 3-space and an embedded curve $\gamma$ lying on one side of $P$ such that any least area disk $D$ which solves the Plateau problem for $\gamma$ must intersect $P$.

Proof. We will present the proof in two steps. 


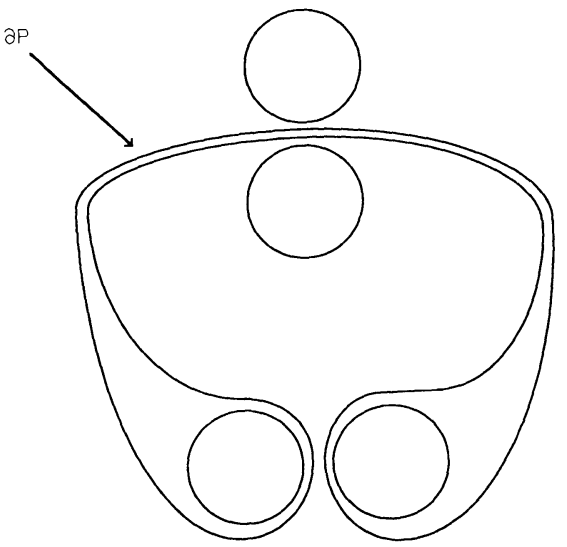

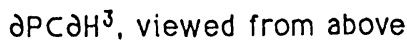

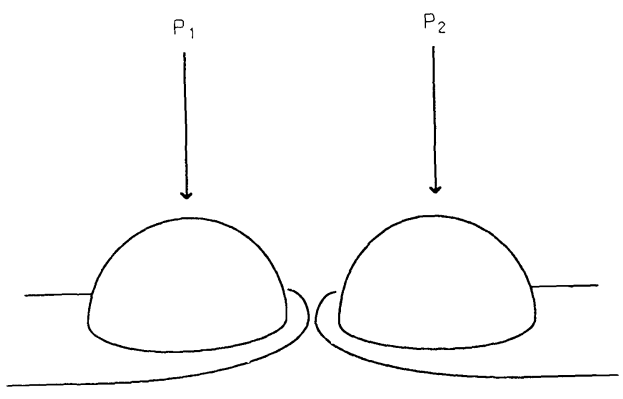

dP running between $P_{1}$ and $P_{2}$

Figure 1

$\partial P$ in the upper half-space model of $H^{3} . P$ misses the totally geodesic planes whose ideal boundaries are round circles.

Step 1. Construction of a least area plane in $H^{3}$ which is not area minimizing. We present a simple construction of a least area plane in hyperbolic 3-space $H^{3}$, based on an example in [A]. Consider the upper half-space model of $H^{3}$. An annulus is said to be least area if there is no other annulus with the same boundary which has less area. Note that this implies that the sum of the areas of two least area disks with the same boundary as the annulus is not less than the area of the annulus, since we can tube these two disks together with a zero area tube to form an annulus.

On the ideal boundary of $H^{3}$ we construct a smooth embedded curve which runs between two pairs of round circles, as in Figure 1. Any simple smooth curve on $\partial H^{3}$ is the ideal boundary of an embedded least area plane $P$ properly embedded in $H^{3}$ [A]. If two totally geodesic planes $P_{1}$ and $P_{2}$ are sufficiently close in $H^{3}$ then we can find on them curves $a_{1}$ and $a_{2}$ which cobound an embedded least area annulus $A$ whose area is strictly less than the sum of the areas of the two totally geodesic disks that they bound on $P_{1} \cup P_{2}[\mathbf{M}-\mathbf{Y}]$. Note that $P$ cannot intersect the hyperplanes in $H^{3}$ corresponding to each of the circles depicted in Figure 1, since if it did we could find a hyperplane with boundary a smaller concentric circle which touches $P$ without crossing it, violating the maximal principle for minimal surfaces. With $P_{1}$ and $P_{2}$ and $A$ as above, a pair of parallel components $d_{1}$ and $d_{2}$ of $P \cap A$ cobound a least area subannulus 
$A^{\prime}$ of $A$. A subannulus of a least area annulus is also least area. In fact the area of $A^{\prime}$ is strictly less than the sum of the area of the least area disks that its boundary curves bound, as otherwise the two disks together with $A-A^{\prime}$ form a pair of disks bounding $a_{1} \cup a_{2}$ with area at most equal to the area of $A$ and this area could be decreased by smoothing off the corner where the disks join $A-A^{\prime}$, contradicting the assumption that $A$ is least area. So $P$ is not area minimizing.

Step 2. Construction of the curve $\gamma \subset H^{3}$. We now proceed to construct an embedded curve $\gamma$ which will have the property that any disk of least area bounded by $\gamma$ must intersect $P$. The curve is indicated in Figure $2 b$.

Let $D_{1}$ denote a least area disk with boundary $a_{1} \subset P_{1}, D_{2}$ a least area disk with boundary $a_{2} \subset P_{2}$ and recall that $A$ is a least area annulus cobounding $a_{1}$ and $a_{2}$. Then by our choice of $P_{1}$ and $P_{2}$ we have that $\operatorname{Area}\left(D_{1}\right)+\operatorname{Area}\left(D_{2}\right)-\varepsilon>\operatorname{Area}(A)$ for some positive constant $\varepsilon$. Fix an orientation on $A$ and orient $a_{1}$ and $a_{2}$ as the boundary of $A$. Let $b$ be a simple arc on the same side of $P$ as $a_{1}$ and $a_{2}$ which joins $a_{1}$ to $a_{2}$, let $a$ be a simple arc on $A$ joining the endpoint of $b$ on $a_{1}$ to the endpoint of $b$ on $a_{2}$ and let $E$ be a disk in $H^{3}$ bounded by $a \cup b$.

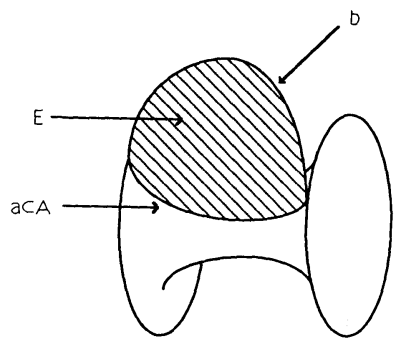

Figure 2a

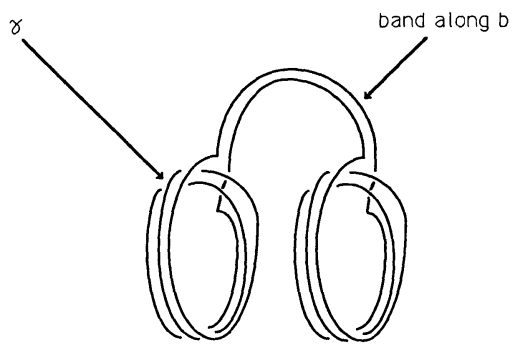

FIGURE $2 b$

Pick a large integer $k$ such that $k \varepsilon>2 \operatorname{Area}(E)$ and let the curve $\gamma^{\prime}$ be obtained by running $k$ times around $a_{1}$, once along $b, k$ times around $a_{2}$ and back along $b$. The singular curve $\gamma^{\prime}$ bounds a disk $D^{\prime}$ formed by taking a copy of each of the $k$-fold branch covers of $D_{1}$ and $D_{2}$ and banding them together along a zero width band running along $g$. $D^{\prime}$ misses $P$. Area $\left(D^{\prime}\right)=k$ Area $\left(D_{1}\right)+k \operatorname{Area}\left(D_{2}\right)$, and since $D_{1}$ and $D_{2}$ are totally geodesic, intersection theory implies that no disk bounded by $\gamma^{\prime}$ and missing $P$ has less area. So $D^{\prime}$ has least area among disks in the complement of $P$. But $\gamma^{\prime}$ bounds a disk $D$ 
meeting $P$ formed by taking the $k$-fold cover of the annulus $A$, cutting it open along the arc $a$ to get a disk, and gluing on two copies of $E$ along $a$. The area of this disk is precisely $k \operatorname{Area}(A)+2 \operatorname{Area}(E)$. So $\operatorname{Area}(D)=k \operatorname{Area}(A)+2 \operatorname{Area}(E)<k \operatorname{Area}\left(D_{1}\right)+k \operatorname{Area}\left(D_{2}\right)-$ $k \varepsilon+2 \operatorname{Area}(E)<k \operatorname{Area}\left(D_{1}\right)+k \operatorname{Area}\left(D_{2}\right)-k \varepsilon+k \varepsilon=\operatorname{Area}\left(D^{\prime}\right)$. Any disk in the complement of $P$ has area strictly larger than $\operatorname{Area}\left(D^{\prime}\right)$, but the disk $D$ meeting $P$ has strictly less area. There is a constant $\delta>0$ such that the least area disk $D^{\prime \prime}$ in $H^{3}$ with boundary $\gamma^{\prime}$ has $\operatorname{Area}\left(D^{\prime \prime}\right)<\operatorname{Area}\left(D^{\prime}\right)-2 \delta . \quad \gamma^{\prime}$ can be perturbed slightly to give an embedded curve $\gamma$ missing $P$, and the perturbation can be picked sufficiently small so that $\gamma$ and $\gamma^{\prime}$ cobound an annulus of area less than $\delta$. It then follows that $\gamma$ lies on one side of $P$ and that any least area disk which solves the Plateau problem for $\gamma$ must intersect $P$.

REMARKS. It is not essential that the ambient manifold in which we construct the above example is hyperbolic 3-space. The same construction can be carried out in any manifold where there is a least area plane $P$ for which one can find an annulus mapped properly into the complement of $P$ whose boundary components bound two disjoint disks in $P$ with area greater than the area of the annulus. Such a least area plane cannot be found in $R^{3}$, or in a manifold of nonnegative scalar curvature [S-Y], so there are some restrictions on the ambient manifold.

This example also provides an immersed curve lying on a least area plane which bounds a least area disk not on the plane. Note that one can solve the existence problem for least area disks with singular curves as boundaries $[\mathbf{H}]$. For embedded curves, the least area disk lies on the plane by definition. Singular curves exhibit a markedly different behavior.

\section{REFERENCES}

[A] M. Anderson, Complete minimal hypersurfaces in hyperbolic $n$-manifolds, Comment. Math Helvetici, 58 (1983), 264-290.

[F-H-S] M. Freedman, J. Hass and P. Scott, Least area incompressible surfaces in 3-manifolds, Invent. Math., 71 (1983), 609-642.

[H] J. Hass, Singular curves and the Plateau problem, Intl. J. Math., 2 (1991), $1-16$.

[M-Y] W. H. Meeks III and S. T. Yau, Topology of three dimensional manifolds and the embedding theorems in minimal surface theory, Annals of Math., 112 (1980), 441-484. 
[P] C. D. Papakyriakopoulos, On Dehn's lemma and the asphericity of knots, Annals of Math., 66 (1957), 1-26.

[S-Y] R. Schoen and S. T. Yau, Complete three dimensional manifolds with positive Ricci curvature and scalar curvature, Seminar on Differential Geometry, Annals of Math. Studies, 102 (1982), 209-228.

Received September 5, 1990. Partially supported by NSF grant DMS-8823009 \& a grant from the Sloan Foundation.

INSTITUTE FOR ADVANCED STUDY

PRINCETON, NJ 08540

Current address: University of California

Davis, CA 95616

jhass@ucdavis.edu 



\title{
PACIFIC JOURNAL OF MATHEMATICS EDITORS
}

\author{
V. S. VARADARAJAN \\ (Managing Editor) \\ University of California \\ Los Angeles, CA 90024-1555-05 \\ Herbert Clemens \\ University of Utah \\ Salt Lake City, UT 84112 \\ F. Michael Christ \\ University of California \\ Los Angeles, CA 90024-1555 \\ THOMAS ENRIGHT \\ University of California, San Diego \\ La Jolla, CA 92093
}

Nicholas ERcolani

University of Arizona

Tucson, AZ 85721

R. FINN

Stanford University

Stanford, CA 94305

VAUGHAN F. R. JoNeS

University of California

Berkeley, CA 94720

C. C. MOORE

University of California

Berkeley, CA 94720

MARTIN SchaRLEMANN

University of California

Santa Barbara, CA 93106

HAROLd StaRK

University of California, San Diego

La Jolla, CA 92093

SteVen Kerckhoff

Stanford University

Stanford, CA 94305

\section{ASSOCIATE EDITORS}

\begin{tabular}{|c|c|c|c|c|}
\hline R. ARENS & $\begin{array}{l}\text { E. F. BECKENBACH } \\
(1906-1982)\end{array}$ & NeumanN & $\begin{array}{c}\text { F. WoLF } \\
(1904-1989)\end{array}$ & K. YoshidA \\
\hline \multicolumn{5}{|c|}{ SUPPORTING INSTITUTIONS } \\
\hline \multicolumn{2}{|c|}{ JNIVERSITY OF ARIZONA } & \multicolumn{3}{|c|}{ UNIVERSITY OF OREGON } \\
\hline \multicolumn{2}{|c|}{ UNIVERSITY OF BRITISH COLUMBIA } & \multicolumn{3}{|c|}{ UNIVERSITY OF SOUTHERN C } \\
\hline \multicolumn{2}{|c|}{ CALIFORNIA INSTITUTE OF TECHNOLOGY } & \multirow{2}{*}{\multicolumn{3}{|c|}{ STANFORD UNIVERSITY }} \\
\hline \multicolumn{2}{|c|}{ UNIVERSITY OF CALIFORNIA } & & & UNIVERSITY OF HAWAII \\
\hline \multicolumn{2}{|c|}{ MONTANA STATE UNIVERSITY } & \multicolumn{3}{|c|}{ UNIVERSITY OF TOKYO } \\
\hline \multicolumn{2}{|c|}{ UNIVERSITY OF NEVADA, RENO } & \multicolumn{3}{|c|}{ UNIVERSITY OF UTAH } \\
\hline \multicolumn{2}{|c|}{ NEW MEXICO STATE UNIVERSITY } & \multicolumn{3}{|c|}{ DN STATE UNIVERSITY } \\
\hline \multicolumn{2}{|c|}{ OREGON STATE UNIVERSITY } & UNIVER & OF WASHIN & \\
\hline
\end{tabular}




\section{Pacific Journal of Mathematics}

Vol. 152, No. $1 \quad$ January, 1992

B. V. Rajarama Bhat, On a characterization of velocity maps in the space of observables .......................................

John David Brillhart, Note on the discriminant of certain cyclotomic period

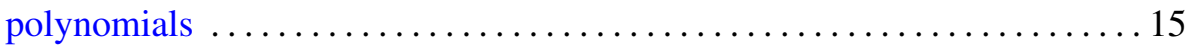

Steven R. Costenoble and Stefan Waner, The equivariant Thom

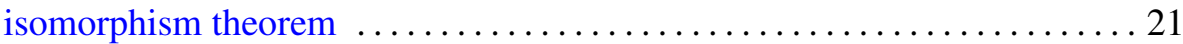

Karl Heinz Dovermann and Dong Youp Suh, Smith equivalence for finite

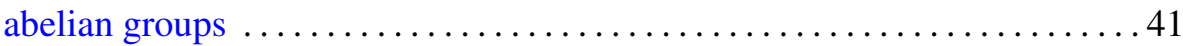

Klaus-Jochen Engel, On singular perturbations of second order Cauchy

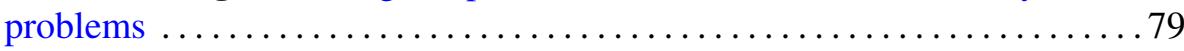

Angel Ferrandez and Pascual Lucas, On surfaces in the 3-dimensional

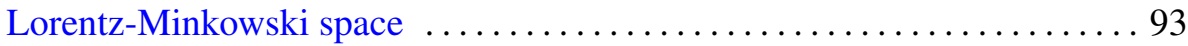

Brian Hartley, A general Brauer-Fowler theorem and centralizers in locally

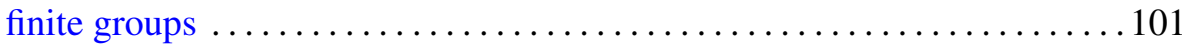

Joel Hass, Intersections of least area surfaces .................... 119

Edward Kissin, Indices of unbounded derivations of $C^{*}$-algebras $\ldots \ldots \ldots 125$

Erhard Luft and Denis Karmen Sjerve, On regular coverings of

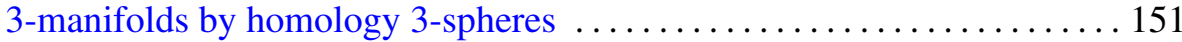

Daniel Pecker, Sur la projection de variétés algébriques réelles .......... 165

Claus Scheiderer, Some remarks on orderings under finite field

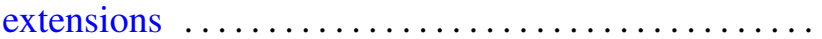

Fernando Serrano, Elliptic surfaces with an ample divisor of genus two 